学位論文

Pulse oximetry-derived pleth variability index can predict dexmedetomidine-induced changes in blood pressure in spontaneouslybreathing patients

（指先部潅流指数を用いたデクスメデトミジン投与における血圧変動予測）

佐藤 慎

(Takayuki Kunisawa, Atsushi Kurosawa, Tomoki Sasakawa) 


\title{
Pulse oximetry-derived pleth variability index can predict dexmedetomidine-induced changes in blood pressure in spontaneously

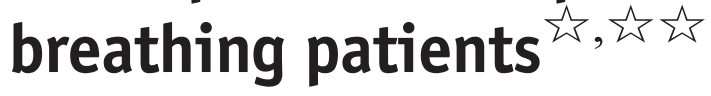

\author{
Makoto Sato MD (Clinical Fellow) ${ }^{\star}$, Takayuki Kunisawa MD, PhD (Professor), \\ Atsushi Kurosawa MD (Associate Professor), \\ Tomoki Sasakawa MD, PhD (Senior Lecturer)
}

Department of Anesthesiology and Critical Care Medicine, Asahikawa Medical University, 2-1-1-1 Midorigaoka Higashi, Asahikawa, Hokkaido 788-510, Japan

Received 10 March 2016; revised 27 April 2016; accepted 2 May 2016

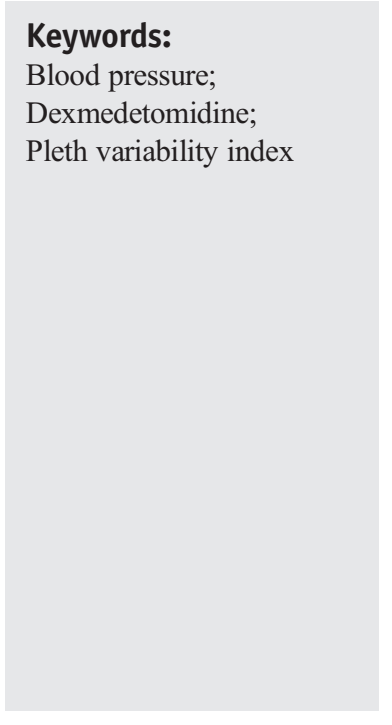

\begin{abstract}
Study objective: Hypertension or hypotension in patients receiving continuous infusions of dexmedetomidine (DEX) is often due to changes in vascular resistance caused by $\alpha_{2}$ receptor stimulation. We investigated whether baseline perfusion index (PI) and pleth variability index (PVI), derived from pulse oximetry readings, could predict DEX-induced changes in the hemodynamic status in spontaneously breathing patients. Design: Observational study.

Setting: Operating room.

Patients: Patients (American Society of Anesthesiologists performance status 1 or 2) scheduled to undergo lower extremity or abdominal procedures under regional anesthesia were approached.

Interventions: The PI and PVI were set as baseline upon arrival in theater and were then measured at 2.5minute intervals. Upon attaining stable hemodynamic status under spontaneous breathing, intravenous administration of DEX was initiated at $6 \mu \mathrm{g} \mathrm{kg}^{-1} \mathrm{~h}^{-1}$ for 10 minutes, followed by continuous infusion at $0.6 \mu \mathrm{g} \mathrm{kg}^{-1} \mathrm{~h}^{-1}$.

Measurements: Blood pressure, heart rate, PI, and PVI were measured. Hypertension was defined as an increase in systolic blood pressure (SBP) $>15 \%$ and hypotension as a decrease in SBP $<15 \%$ from baseline. Main results: Baseline PI and PVI correlated with the degree of change in SBP. The maximum percentage increase as well as the maximum percentage of decrease in SBP from baseline correlated with baseline PI $(r=0.418[P=.005]$ and $r=0.507[P<.001]$, respectively) and PVI $(r=-0.658[P<.001]$ and
\end{abstract}

\footnotetext{
is Conflicts of interest: The authors declare no conflicts of interest.

is is Disclosures: The paper's abstract was published in “Anesthesiology ${ }^{\circledR} 2015$ Annual Meeting Abstract Website": Hyperlink: http://www.asaabstracts.com/ strands/asaabstracts/abstract.htm;jsessionid=68A51D2FD1C16AF64D06A3D42EAAF23A?year=2015\&index=8\&absnum=3065

* Corresponding author: Makoto Sato, MD, Department of Anesthesiology and Critical Care Medicine, Asahikawa Medical University, 2-1-1-1 Midorigaoka Higashi, Asahikawa, Hokkaido 788-510, Japan. Tel.: +81 16668 2583; fax: +81 166682589 .

E-mail addresses: satomako@asahikawa-med.ac.jp (M. Sato), taka.kunisawa@nifty.ne.jp (T. Kunisawa),work96@mac.com (A. Kurosawa), sasakawa@asahikawa-med.ac.jp (T. Sasakawa).
} 
$r=-0.438[P=.003]$, respectively). PVI $<15$ identified DEX-induced hypertension (sensitivity 94\%, specificity $85 \%$ ) and PVI $>16$ identified DEX-induced hypotension (sensitivity 83\%, specificity 64\%).

Conclusions: PVI may predict DEX-induced changes in blood pressure in spontaneously breathing patients. (C) 2016 Elsevier Inc. All rights reserved.

\section{Introduction}

Hypertension and hypotension in patients receiving continuous infusions of dexmedetomidine (DEX) are often the consequence of increased or decreased vascular resistance, respectively, due to $\alpha_{2}$ receptor stimulation. These conflicting hemodynamic changes may be induced by initial loading doses of DEX at various time points and lead to a critical condition even in nonintubated patients, although an initial loading is needed for rapid onset of anesthesia. If the changes in blood pressure could be predicted, assessment and treatment of hypertension and hypotension caused by DEX would be possible.

A previous study has demonstrated that the condition of patients determines which site (central nervous system or peripheral vessels) is most affected by DEX administration [1]. For example, systolic blood pressure (SBP) can decrease because of the sedative effect of DEX in patients who are in an emergency condition and who are experiencing pain or stress. In contrast, SBP can increase because of a peripheral vasoconstrictive effect in patients who have received sufficient amounts of anesthetics. However, no useful and objective indicators of DEX-induced hemodynamic changes in conscious patients have been found to date.

The perfusion index (PI) and pleth variability index (PVI), derived from pulse oximetry, have been used to evaluate peripheral perfusion dynamics from changes in peripheral vascular tone. The PI is defined as the ratio between constant absorption and pulsatile absorption and reflects the amplitude of the plethysmographic waveform. PVI can automatically detect the maximum and minimal PI value over a sufficient period of time. Evaluation of patients' peripheral vascular status may be helpful to predict DEX-induced hemodynamic changes. Moreover, because the probe for pulse oximetry is attached only to a finger and is therefore noninvasive, it is helpful, particularly in conscious patients where arterial and central venous catheters are typically not used to measure hemodynamic parameters.

This study was aimed to examine whether baseline PI and PVI could be useful to predict DEX-induced hemodynamic changes in spontaneously breathing patients. We aimed to determine optimal cutoff points for PI and PVI to predict DEXinduced hypertension and hypotension.

\section{Materials and methods}

\subsection{Study population}

This study was approved by the Institutional Ethics Committee of Asahikawa Medical University, Asahikawa, Japan, and was performed according to World Medical Association Ethical Principles for Medical Research Involving Human Subjects. We investigated patients attending the Asahikawa Medical University Hospital from February 2014 to February 2015. Forty-two patients scheduled to undergo lower extremity or abdominal procedures under regional anesthesia were approached prior to anesthesia; patients provided oral and written informed consent to enter in this study. The exclusion criteria were an American Society of Anesthesiologists (ASA) classification of III or greater, age below 18 years, pregnancy, inability to give informed consent, or the presence of severe arteriosclerosis obliterans, arrhythmia (atrial fibrillation), heart failure (ejection fraction $<40 \%$ ), renal failure (plasma creatinine level $>2 \mathrm{mg} / \mathrm{dL}$ ), and previous use of medications involving $\alpha$ - or $\beta$-adrenergic agonists or antagonists.

\subsection{Hemodynamic status monitoring}

Premedication was not needed. Upon arrival in the operating room, each patient was placed in the supine position and was given an intravenous infusion of bicarbonate or acetate Ringer's solution, at 100 to $200 \mathrm{~mL} / \mathrm{h}$. Standard monitoring by means of electrocardiography, automated noninvasive blood pressure (NIBP) monitoring, and pulse oximetry was carried out. The automated NIBP device cuff was attached to the right arm, and SBP and diastolic blood pressure were measured at 2.5-minute intervals. The probe of the pulse oximeter (Masimo Radical-7; Masimo Corp, Tokyo, Japan) was attached to the left fourth finger. Baseline NIBP, heart rate (HR), PI, and PVI were monitored in the supine position. The attending anesthesiologists were blinded to the values of PI and PVI.

When hemodynamic status had stabilized under spontaneous breathing, DEX was administered at $6 \mu \mathrm{g} \mathrm{kg}^{-1} \mathrm{~h}^{-1}$ for $10 \mathrm{mi}-$ nutes and then modulated to $0.6 \mu \mathrm{g} \mathrm{kg}^{-1} \mathrm{~h}^{-1}$. At least $20 \mathrm{mi}-$ nutes after the initiation of DEX administration, appropriate regional anesthesia, according to the surgical site (intrathecal anesthesia, lumbar plexus block, femoral nerve block, or sciatic nerve block), was administered by the anesthesiologist.

NIBP, HR, PI, and PVI were recorded for 20 minutes during administration of DEX. Hypertension was defined as an increase in SBP to a value greater than $15 \%$ or $20 \mathrm{~mm} \mathrm{Hg}$ from baseline and hypotension as a decrease in SBP to a value of less than $15 \%$ or $20 \mathrm{~mm} \mathrm{Hg}$ from baseline, as defined in a previous study [2]. When SBP increased more than 30\% from baseline, $0.5 \mathrm{mg}$ nicardipine was dispensed intravenously, and when SBP decreased more than $30 \%$ from baseline, $5 \mathrm{mg}$ ephedrine was dispensed intravenously as a rescue medication. We dispensed $0.5 \mathrm{mg}$ atropine intravenously if severe 
bradycardia (HR $<40$ beats/min) occurred. Oxygen was given if the arterial oxyhemoglobin saturation, as determined by pulse oximetry, decreased to $<93 \%$.

\subsection{Statistical analysis}

Patient population data are presented as means (standard deviation) or medians (range), where appropriate. Because no published reports have included data regarding the strength of the correlation between baseline PI or PVI and the degree of change in SBP from baseline, a correlation hypothesis of coefficient $r=0.42$ was assumed in this study based on our pilot study that showed $r=0.415(P<.001)$ between baseline PI and the change in SBP from baseline. To detect a correlation coefficient at a power of 0.8 and a 2 -tailed $\alpha$ of $.05,42$ patients were required in this study.

To assess the correlation between baseline PI or PVI and the degree of change in SBP from baseline, Spearman rank correlation coefficient was used. To assess the relationship between patient characteristics and baseline parameters with the degree of change in SBP from baseline, multiple linear regression analysis was performed. Backward stepwise procedures were used to select independently associated variables, without multicollinearity among the covariates.

Receiver operating characteristic (ROC) curves for hypertension and hypotension were generated and areas under the ROC curves were calculated to assess the abilities of baseline PI and PVI to predict DEX-induced changes in blood pressure.

Patient population data were compared using the unpaired Student $t$ test, Mann-Whitney $U$ test, or Fisher exact probability test, where appropriate. Perioperative hemodynamic parameters, PI, and PVI were compared using 2-way analysis of variance with the Bonferroni post hoc test. Statistical significance was defined as $P<.05$.

\section{Results}

\subsection{Changes in SBP}

Forty-two patients were included and no patients were excluded from this study or presented an inadequate signal quality for measurement of PI and PVI. Patient characteristics and baseline parameters are presented in Table 1. Preoperative hypertension had been diagnosed in 17 patients (40.5\%), and they had taken 1 or 2 hypertensive medications not involving $\alpha$-adrenergic agonists or antagonists. After administration of DEX, 14 patients (33.3\%) developed hypertension, with a maximum increase in SBP to $35.4 \%$ higher than baseline; 19 patients (45.2\%) developed hypotension, with a maximum decrease in SBP to $45.4 \%$ lower than baseline. One patient developed both hypertension at 10 minutes after administration of DEX and hypotension at 20 minutes after administration of DEX. The median (range) nicardipine requirement was $0(0-2) \mathrm{mg}$, and the ephedrine requirement was $0(0-10) \mathrm{mg}$. Atropine was not administered to any patient.
Table 1 Patient characteristics and baseline parameters

\begin{tabular}{ll} 
Characteristics & \\
\hline Age $(\mathrm{y})$ & $65(29-89)$ \\
Sex $(\mathrm{M} / \mathrm{F})$ & $17 / 25$ \\
Height $(\mathrm{cm})$ & $156.3(9.82)$ \\
Weight $(\mathrm{kg})$ & $58.5(12.0)$ \\
ASA-PS (I/II) & $12 / 30$ \\
Baseline parameters & \\
SBP (mm Hg) & $139(25.9)$ \\
DBP (mm Hg) & $69(16.5)$ \\
HR (beats/min) & $76(13.5)$ \\
PI (\%) & $2.97(2.06)$ \\
PVI (\%) & $17(7.05)$ \\
\hline Data are presented as mean (range) for age and mean (standard deviation) \\
for other parameters. \\
ASA = American Society of Anesthesiologists; PS = performance status; \\
SBP = systolic blood pressure; DBP = diastolic blood pressure; HR = \\
heart rate; PI = perfusion index; PVI = pleth variability index.
\end{tabular}

Table 2 shows correlations between the change in SBP and patient characteristics and baseline parameters. The maximum percentage increase in SBP from baseline, observed $10 \mathrm{mi}-$ nutes after administration of DEX in most cases, was statistically correlated with baseline PI $(r=0.418, P=.005$; Table 2) and PVI $(r=-0.658, P<.001$; Table 2, Fig. 1$)$. The maximum percentage decrease in SBP from baseline, observed 20 minutes after administration of DEX in most cases, was also correlated with baseline PI $(r=0.507, P<.001$; Table 2), PVI $(r=-0.438, P=.003$; Table 2$)$, and baseline SBP $(r=-0.345, P=.025$; Table 2).

Multiple linear regression analysis revealed that baseline PVI statistically contributed to the maximum percentage

Table 2 Correlations between the degree of change in systolic blood pressure (SBP) and baseline parameters

\begin{tabular}{|c|c|c|c|c|}
\hline & \multicolumn{2}{|c|}{$\begin{array}{l}\text { Maximum \% SBP } \\
\text { increase }\end{array}$} & \multicolumn{2}{|c|}{$\begin{array}{l}\text { Maximum \% SBP } \\
\text { decrease }\end{array}$} \\
\hline & $r$ & $P$ & $r$ & $P$ \\
\hline \multicolumn{5}{|l|}{ Characteristics } \\
\hline Age (y) & -0.056 & .724 & -0.214 & .174 \\
\hline Sex & -0.212 & .177 & -0.15 & .343 \\
\hline Height (cm) & 0.115 & .469 & 0.183 & .245 \\
\hline Weight (kg) & 0.138 & .383 & 0.267 & .087 \\
\hline ASA-PS & -0.544 & .732 & -0.148 & .35 \\
\hline \multicolumn{5}{|c|}{ Baseline parameters } \\
\hline $\mathrm{SBP}(\mathrm{mm} \mathrm{Hg})$ & -0.205 & .192 & -0.345 & .025 \\
\hline DBP (mm Hg) & -0.137 & .387 & -0.071 & .657 \\
\hline HR (beats/min) & -0.136 & .391 & -0.144 & .362 \\
\hline PI (\%) & 0.418 & .005 & 0.507 & $<.001$ \\
\hline PVI (\%) & -0.658 & $<.001$ & -0.438 & .003 \\
\hline
\end{tabular}

Data are determined by the Spearman rank correlation.

ASA = American Society of Anesthesiologists; PS = performance status; $\mathrm{DBP}=$ diastolic blood pressure; $\mathrm{HR}=$ heart rate; $\mathrm{PI}=$ perfusion index $\mathrm{PVI}=$ pleth variability index. 


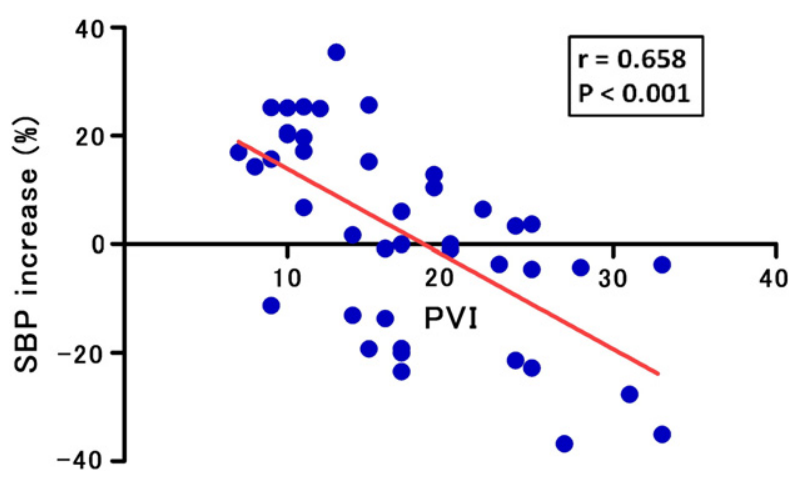

Fig. 1 Correlation between baseline PVI and SBP increase. Correlation between baseline pleth variability index (PVI) and each maximum percentage increase in systolic blood pressure $(\mathrm{SBP})$ from baseline. SBP increase $=([$ highest SBP - baseline SBP $] \times$ baseline SBP -1$)$. The solid line represents the linear regression line.

increase in SBP from baseline (predicted maximum percentage increase in SBP from baseline $=30.514-[1.659 \times$ baseline PVI]; $R^{2}=0.35, P=.006$ ), and baseline PI and PVI contributed to each maximum percent decrease in SBP (predicted maximum percentage decrease in SBP from baseline $=-3.396+[2.993 \times$ baseline PI $]-[0.861 \times$ baseline PVI]; $\left.R^{2}=0.24, P=.039\right)$.

\subsection{PVI and DEX-induced hemodynamic changes}

ROC curve analysis confirmed that baseline PVI was appropriate for detecting patients at risk of DEX-induced hypertension or hypotension (area under the curve $[\mathrm{AUC}]=0.931$ $[P<.001]$ and AUC $=0.785[P<.001]$, respectively; Fig. 2). PVI $<15$ identified DEX-induced hypertension with

\section{A}

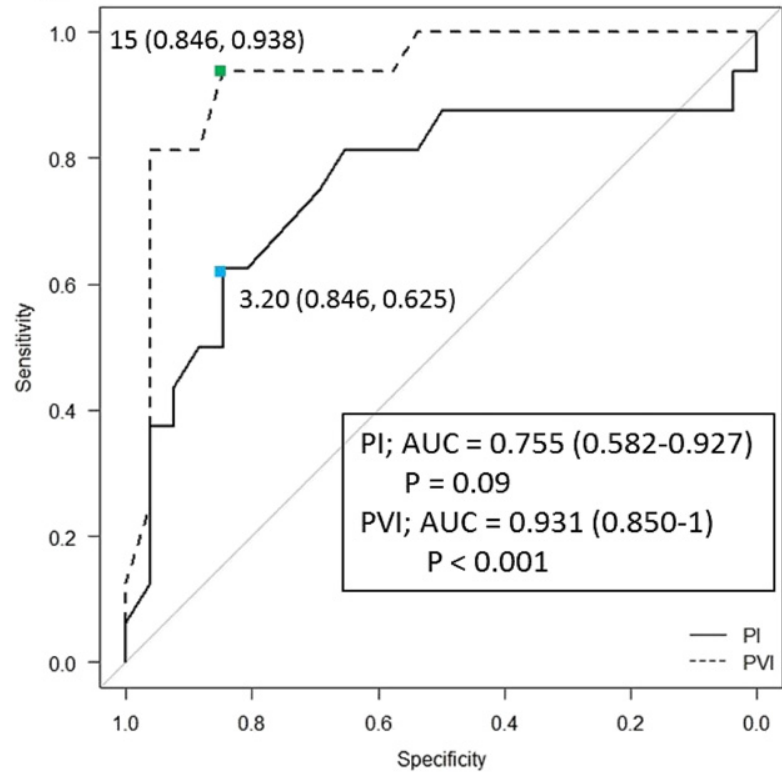

a sensitivity of $94 \%$ and a specificity of $85 \%(95 \%$ confidence interval, 85\%-100\%). PVI > 16 identified DEX-induced hypotension with sensitivity of $83 \%$ and a specificity of $64 \%(95 \%$ confidence interval, 64\%-93\%). Fig. 3 shows the relationship between these cutoff points and the change in SBP. Baseline PVI was correlated with baseline PI ( $r=-0.325, P=.035$; Fig. 3$)$.

Patient characteristics were compared according to the baseline PVI cutoff points of 15 and 16, as determined from the ROC curve analysis. Eighteen patients (42.9\%) had a low baseline PVI (PVI $\leq 15)$. Hypertension was observed in 13 of these 18 patients $(72.2 \%)$ and hypotension in $4(22.2 \%)$. Twenty-four patients $(57.1 \%)$ had a high baseline PVI (PVI $\geq 16)$. Hypertension was observed in $1(4.2 \%)$ of these 24 patients, and hypotension in $14(58.3 \%)$. The patient groups (low and high PVI) were not different with respect to age, height, weight, ASA classification, preoperative laboratory data, and fasting time.

\section{B}

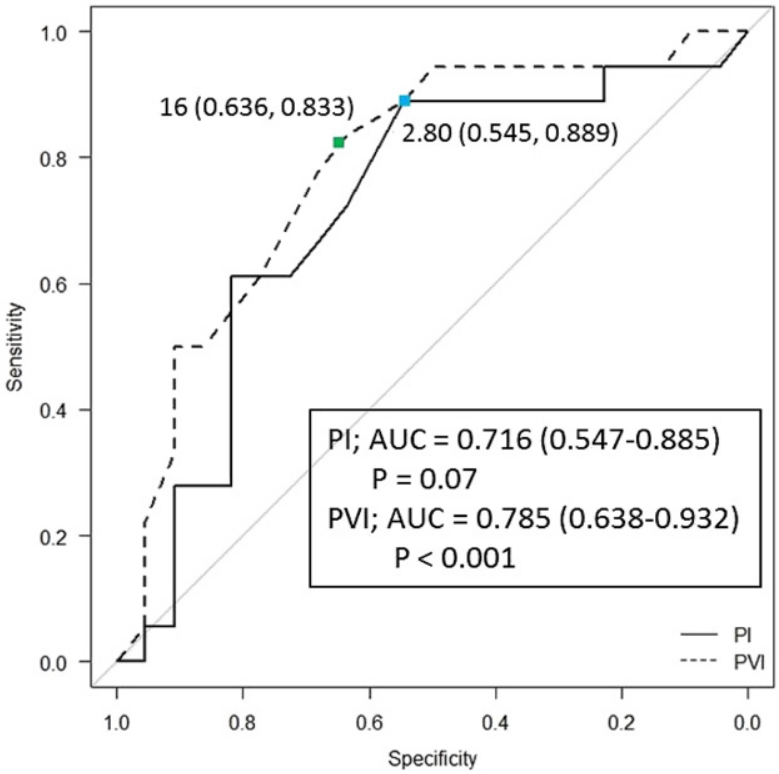

Fig. 2 Receiver operating characteristic (ROC) curves for baseline PI and PVI. ROC curves for baseline perfusion index (PI) and pleth variability index (PVI) to detect dexmedetomidine-induced hypertension (A) and hypotension (B). AUC, area under the ROC curve, with $95 \%$ confidence intervals. 


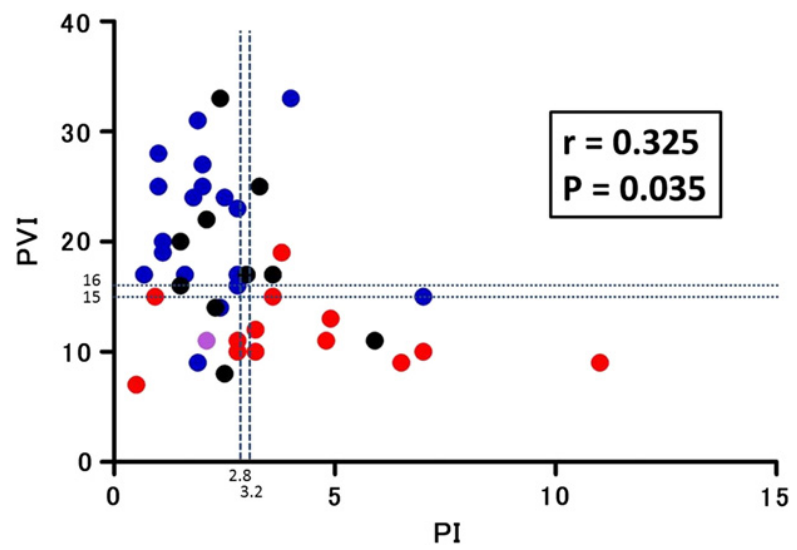

Fig. 3 Scatter diagram graph of patients about PI and PVI. Scatter diagram of baseline perfusion index (PI) and pleth variability index (PVI) of patients. Red dots indicate patients who developed hypertension (an increase in SBP $>15 \%$ from baseline) during administration of dexmedetomidine (DEX). Blue dots indicate patients who developed hypotension (a decrease in SBP $<15 \%$ from baseline) during administration of DEX. The purple point indicates a patient who developed both hypertension and hypotension during administration of DEX. The black point indicates patients who developed neither hypertension nor hypotension during administration of DEX.

Hemodynamic changes in both groups are presented in Fig. 4A-E. Although SBP decreased significantly 17.5 and 20 minutes after administration of DEX in patients with a high baseline PVI (both $P<.05$; Fig. 4A), patients with a low baseline PVI had a transiently higher value in SBP 10 minutes after administration of DEX $(P<.05$; Fig. 4A). Baseline PI did not differ significantly between patients with a high baseline PVI and those with a low baseline PVI $(P>.05$; Fig. 4D). The change in PVI in patients with a low baseline PVI was not significant throughout administration of DEX. However, PVI in patients with a high baseline PVI was markedly decreased at $5,7.5,10,12.5,15,17.5$, and 20 minutes after administration of DEX (all $P<.05$; Fig. 4E).
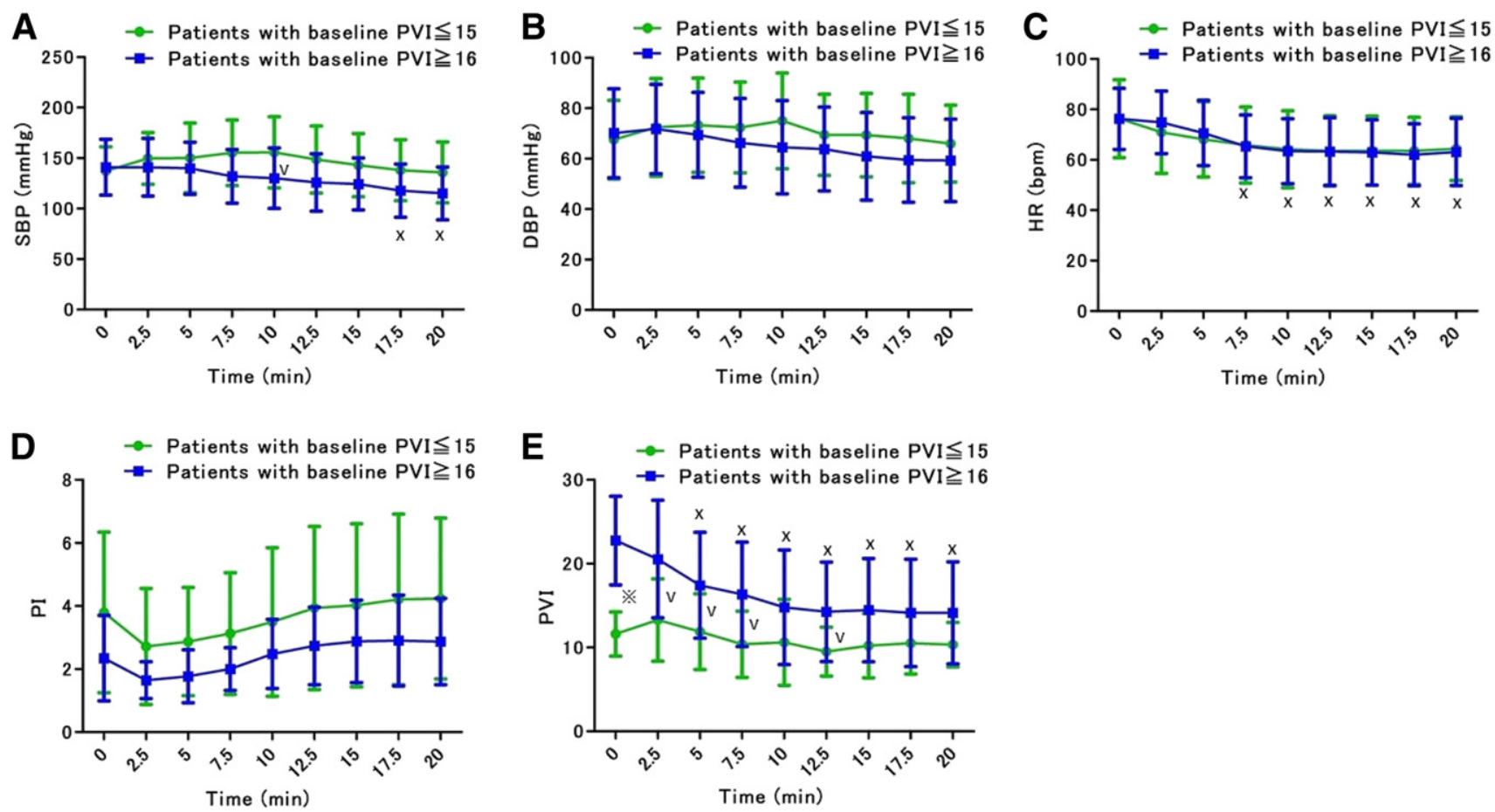

Fig. 4 Summary of the changes in parameters. Summary of the changes in systolic blood pressure (SBP; A), diastolic blood pressure (DBP; B), heart rate (HR; C), perfusion index (PI; D), and pleth variability index (PVI; E) from baseline through 20 minutes after administration of dexmedetomidine. Data are presented as means (standard deviation). x: $P<.05$ vs the group's baseline value; v: $P<.05$ for patients with a high baseline PVI (PVI $\geq 16)$ vs those with a low baseline PVI (PVI $\leq 15)$ during administration of dexmedetomidine. 
DEX, which has selective $\alpha_{2}$ adrenergic properties, could also induce a transient systemic hypertensive reaction [3]. $\alpha_{2}$ Receptors are distributed widely, not only in the central nerve system but also in the peripheral vasculature [4-6]. Blood pressure decreases when DEX acts on the central nerve system, but can also increase because of stimulation of postsynaptic $\alpha_{2}$ receptors in peripheral blood vessels, which has vasoconstrictive effects [7]. Kunisawa et al [1] have reported that blood pressure can decrease in patients whose peripheral blood vessels were sufficiently constricted due to hypovolemia or pain; in contrast, the vasoconstrictive effect was pronounced in patients who had received sufficient anesthetic agents, because the sedative effect was masked. A patient's sympathetic activity before infusion of DEX could predict DEX-induced blood pressure changes; therefore, devices capable of objectively monitoring these changes are needed.

PI and PVI, determined by pulse oximetry, have been used to evaluate peripheral perfusion dynamics. PI depends on vasomotor tone [8,9], and its changes are induced by pulsatile and nonpulsatile changes in arterial and venous blood flow that originate from variations in the vasomotor tone of the blood vessel wall muscles under various situations $[10,11]$. A previous study has shown that an increase in PI was an earlier and more sensitive indicator of the progression of epiduralinduced sympathectomy than was an increase in peripheral temperature [12]. Others have shown that a decrease in PI was a valuable adjunctive diagnostic tool for early detection of a clinically significant central hypovolemic state, prior to the onset of cardiovascular decompensation, in healthy volunteers [13]. Toyama et al [14] reported that a high baseline PI, rather than a change in PI, was associated with profound hypotension during spinal anesthesia for caesarean delivery. PVI is automatically and continuously calculated from the maximal and minimal PI over a sufficient period of time to include at least one complete respiratory cycle and reflects respiratory variations in the pulse oximeter waveform amplitude. Some studies have demonstrated that PVI has a high sensitivity and specificity for predicting fluid responsiveness in mechanically ventilated patients during general anesthesia [15]. Although the reliability of PVI in spontaneous patients still needs to be confirmed, others have demonstrated that PVI is a valuable tool for detecting hemodynamic changes induced by passive leg raising in spontaneously breathing volunteers [16].

Based on these findings, we hypothesized that the relationship between the baseline vasomotor tone and DEX-induced blood pressure changes could be assessed by PI and PVI values in spontaneously breathing patients. To verify our hypothesis, we investigated whether baseline PI and PVI correlated with the degree of change in SBP after administration of DEX and whether baseline PI and PVI could predict a patient's risk for hypertension or hypotension.

We observed a statistical correlation between baseline PI or PVI and the degree of change in SBP from baseline, after administration of DEX, in spontaneously breathing patients. Although there were not clinically significant contributions presented by some of the low $r$ values, baseline PVI had a good contributions to increase in SBP at 10 minutes after administration of DEX. The degree of hypertension was larger and the degree of hypotension was smaller in patients with a low baseline PVI $(\mathrm{PVI} \leq 15)$ than in those with a high baseline PVI (PVI $\geq 16$ ). The higher baseline PVI and/or lower baseline PI in our study may have reflected a relative hypovolemic state and a higher peripheral vascular tone secondary to perioperative stress.

Another noteworthy finding was that a lower baseline PVI and/or a higher baseline PI could predict DEX-induced hypertension in conscious patients. Without anesthetic, SBP may increase after administration of DEX if patients are in a euvolemic state and have sufficient peripheral intravascular volume. We therefore suggest that patients with a lower baseline PVI and a higher baseline PI had a lower peripheral vascular tone and were at higher risk of developing DEX-induced hypertension, because it would have a more pronounced vasoconstrictive effect.

As described in this study, PVI could be a more useful tool than PI as a peripheral baseline perfusion value. Relative to PI, which shows nearly real-time values, the PVI calculation measures changes in PI values over a period of time ( 60 seconds in this study), which can incorporate one or more complete respiratory cycles; hence, PVI values have high repeatability over a particular time period within the same individual and can be useful in spontaneously breathing conscious patients.

The present study has some limitations. First, we did not insert arterial and central venous catheters to measure hemodynamic parameters, such as stroke volume, cardiac output, systemic vascular resistance, and peripheral temperature. However, these are not generally performed in procedures requiring regional anesthesia. Second, the PI value is greatly and easily affected by factors such as stressful and uneasy situations that can cause sympathetic activation (which in turn causes peripheral vasoconstriction), respiratory status, temperature, and movement of the patient. Although the pulse oximeter used in this study is capable of monitoring peripheral perfusion even in critically ill patients, these factors may affect the sensitivity and specificity of preoperative PI and PVI for predicting DEX-induced changes in blood pressure. Third, although baseline PI was correlated with baseline PVI, we could not identify whether patients with a higher baseline PVI and a lower baseline PI were experiencing hypovolemic states, pain, or perioperative stress. Objective stress assessment, such as measurement of stress hormones, was not performed. Fourth, the study population was limited to patients with ASA classifications of I or II. Further studies that include severely ill patients in intensive care units are required to determine the factors that influence the effect of DEX and to establish preventative measures in patients who are predicted to develop hypertension or hypotension during administration of DEX.

In conclusion, baseline PI and PVI, measured by pulse oximetry, statistically correlate with the degree of change in blood pressure during administration of DEX in spontaneously breathing patients. Baseline PVI cutoff points of 15 and 16 could be used to identify patients at risk for DEX-induced 
hypertension and hypotension, respectively. Baseline PVI may be a useful predictor of changes in blood pressure during DEX infusion for sedation of ASA classification I and II patients in clinical practice.

\section{Acknowledgment}

We thank the nursing staff of Asahikawa Medical University Hospital for their assistance during the study and Device Technologies for their technical support.

\section{References}

[1] Kunisawa T, Kawata D, Sugawara A, Iwasaki H. Evaluation of the effect of a loading of dexmedetomidine on blood pressure by factor analysis. Int J Anesthesiol 2014;33(1) [see also http://ispub.com/IJA/33/1/21370].

[2] Mowafi HA, Ismail SA, Shafi MA, Al-Ghamdi AA. The efficacy of perfusion index as an indicator for intravascular injection of epinephrinecontaining epidural test dose in propofol-anesthetized adults. Anesth Analg 2009;108(2):549-53.

[3] Yun Y, Wang J, Tang RR, Yin XR, Zhou H, Pei L. Effects of an intraoperative dexmedetomidine bolus on the postoperative blood pressure and pain subsequent to craniotomy for supratentorial tumors. J Neurosurg Anesthesiol 2016. http://dx.doi.org/10.1097/ANA.0000000000000283.

[4] Kamibayashi T, Maze M. Clinical uses of alpha2-adrenergic agonists. Anesthesiology 2000;93(5):1345-9.

[5] Kunisawa T, Nagata O, Nagashima M, et al. Dexmedetomidine suppresses the decrease in blood pressure during anesthetic induction and blunts the cardiovascular response to tracheal intubation. J Clin Anesth 2009;21(3):194-9

[6] Kunisawa T, Ueno M, Kurosawa A, et al. Dexmedetomidine can stabilize hemodynamics and spare anesthetics before cardiopulmonary bypass. J Anesth 2011;25(6):818-22.

[7] Masuki S, Dinenno FA, Joyner MJ, Eisenach JH. Selective alpha2adrenergic properties of dexmedetomidine over clonidine in the human forearm. J Appl Physiol 2005;99(2):587-92.

[8] Lima AP, Beelen P, Bakker J. Use of a peripheral perfusion index derived from the pulse oximetry signal as a noninvasive indicator of perfusion. Crit Care Med 2002;30:1210-3.

[9] Lima A, Bakker J. Noninvasive monitoring of peripheral perfusion. Intensive Care Med 2005;31(6):1316-26.

[10] Hales JR, Stephens FR, Fawcett AA, et al. Observations on a new noninvasive monitor of skin blood flow. Clin Exp Pharmacol Physiol 1989;16(5):403-15.

[11] Babchenko A, Davidson E, Ginosar Y, et al. Photoplethysmographic measurement of changes in total and pulsatile tissue blood volume, following sympathetic blockade. Physiol Meas 2001;22(2):389-96.

[12] Ginosar Y, Weiniger CF, Meroz Y, et al. Pulse oximeter perfusion index as an early indicator of sympathectomy after epidural anesthesia. Acta Anaesthesiol Scand 2009;53(8):1018-26.

[13] van Genderen ME, Bartels SA, Lima A, et al. Peripheral perfusion index as an early predictor for central hypovolemia in awake healthy volunteers. Anesth Analg 2013;116(2):351-66.

[14] Toyama S, Kakumoto M, Morioka M, et al. Perfusion index derived from a pulse oximeter can predict the incidence of hypotension during spinal anaesthesia for caesarean delivery. Br J Anaesth 2013;111(2):235-41.

[15] Cannesson M, Desebbe O, Rosamel P, et al. Pleth variability index to monitor the respiratory variations in the pulse oximeter plethysmographic waveform amplitude and predict fluid responsiveness in the operating theatre. Br J Anaesth 2008;101(2):200-6

[16] Keller G, Cassar E, Desebbe O, Lehot J-J, Cannesson M. Ability of pleth variability index to detect hemodynamic changes induced by passive leg raising in spontaneously breathing volunteers. Crit Care 2008;12(2):R37. 